\title{
Once-weekly exenatide in a real-world clinical setting: clinical outcomes and analysis of potential predictors of response
}

María del Mar Roca-Rodríguez ${ }^{1}$, Jose Carlos Fernandez-Garcia ${ }^{1}$, Carmen Maria Cortes-Salazar ${ }^{2}$, Isabel Moya-Carmona ${ }^{3}$, Ana Maria Gómez-Pérez ${ }^{1}$, Isabel Cornejo-Pareja ${ }^{1}$, Araceli Muñoz-Garach ${ }^{1}$, María Molina-Vega ${ }^{1}$, Isabel Mancha-Doblas ${ }^{1}$, Francisco Tinahones ${ }^{1}$

1. Endocrinology Department, Virgen de la Victoria University Hospital, Malaga, Spain.

2. Primary Care Center El Palo, Malaga, Spain.

3. Pharmacy Department. Virgen de la Victoria University Hospital, Malaga, Spain.

\section{Objectives}

- The aim of this study is to evaluate the effectiveness of once-weekly exenatide on metabolic control, weight, blood pressure and lipid profile in obese patients with type 2 diabetes (T2DM), and to assess possible predictive factors of response

\section{Baseline characteristics - 1}

\begin{tabular}{|cc|}
\hline & $\mathrm{n}-31$ \\
\hline Age (years) & $54.4 \pm 10.7$ \\
Women $(\%)$ & 54.8 \\
Weight $(\mathrm{kg})$ & $108.1 \pm 28.8$ \\
BMI $\left(\mathrm{kg} / \mathrm{m}^{2}\right.$ ) & $41.2 \pm 8$ \\
TD2M evolution (years) & $7.9 \pm 6.1$ \\
Hypertension (\%) & 83.8 \\
\hline
\end{tabular}

\section{Baseline antidiabetic treatment}

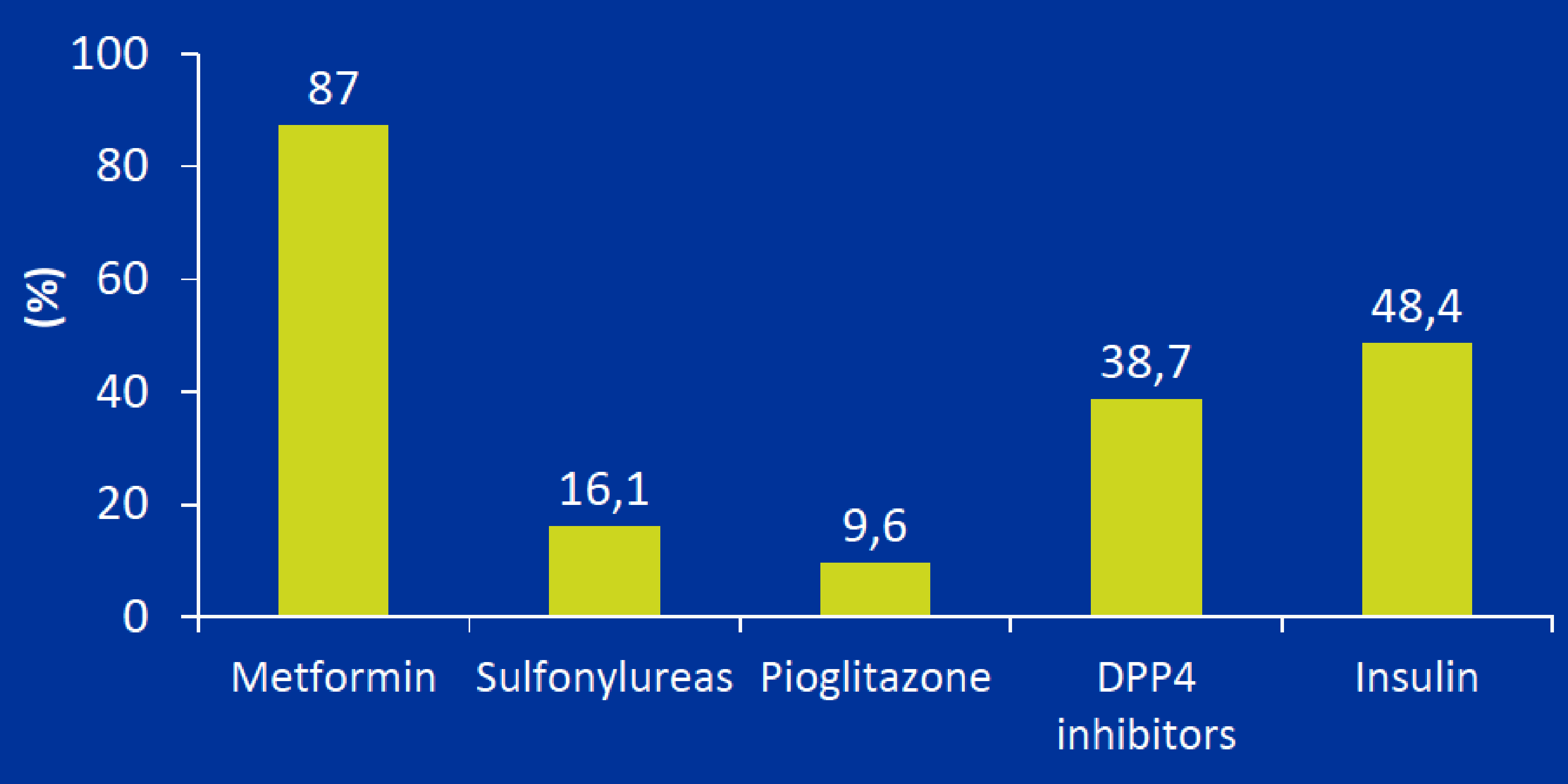

Results - 2

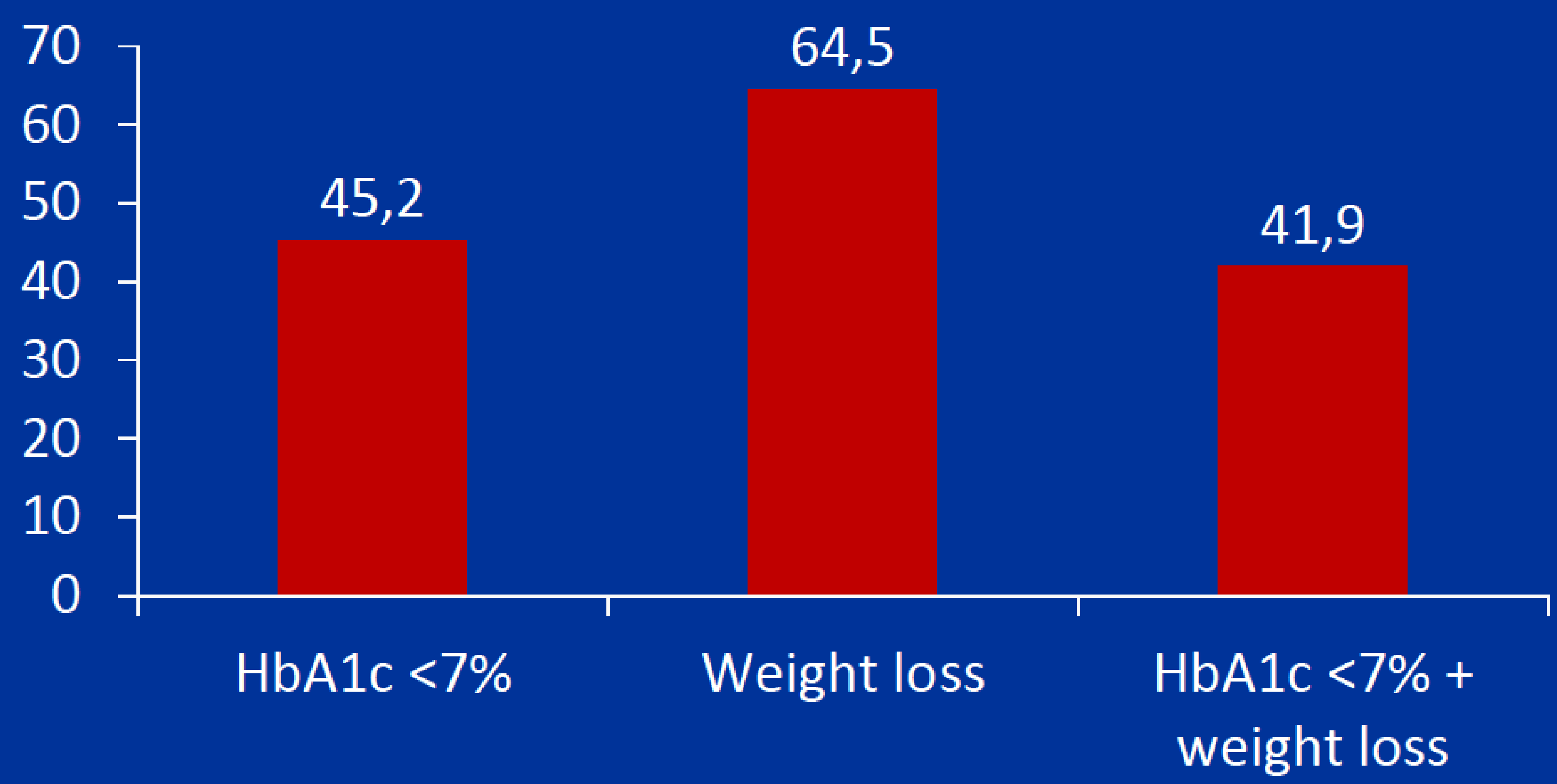
practice. treatment (V1).

weight loss

\section{Material and methods}

- Retrospective observational study, conducted with adult obese (BMI $\geq 30 \mathrm{~kg} / \mathrm{m} 2)$ T2DM subjects that had been initiated once-weekly exenatide following routine clinical

- Anthropometric measures, blood analysis, and blood pressure measures were collected at the initiation of once-weekly exenatide (VO) and after $16 \pm 4$ weeks of

- Predictors of target metabolic response $(\mathrm{HbA} 1 \mathrm{c}<7 \%)$, were evaluated by using a multiple logistic model.

\section{Baseline characteristics - 2}

\begin{tabular}{|cc|}
\hline & \\
\hline & $\mathrm{n}-31$ \\
\hline HbA1c $(\%)$ & $8.6 \pm 1.6$ \\
Cholesterol $(\mathrm{mg} / \mathrm{dl})$ & $206.4 \pm 37$ \\
LDL cholesterol $(\mathrm{mg} / \mathrm{dl})$ & $39.4 \pm 10.7$ \\
Triglycerides $(\mathrm{mg} / \mathrm{dl})$ & $124.3 \pm 35.8$ \\
\hline
\end{tabular}

Results - 1

\begin{tabular}{|cccc|}
\hline & V0 & V1 & $p$ \\
\hline Weight $(\mathrm{kg})$ & $108.1 \pm 28.8$ & $101.3 \pm 22.3$ & $<0.001$ \\
BMI $\left(\mathrm{kg} / \mathrm{m}^{2}\right)$ & $41.2 \pm 8$ & $39.3 \pm 7.3$ & $<0.001$ \\
HbA1c $(\%)$ & $8.6 \pm 1.6$ & $7.3 \pm 1.3$ & $<0.001$ \\
Cholesterol $(\mathrm{mg} / \mathrm{dl})$ & $206.4 \pm 37$ & $181 \pm 41.5$ & 0.002 \\
HDL cholesterol $(\mathrm{mg} / \mathrm{dl})$ & $39.4 \pm 10.7$ & $40.7 \pm 10.3$ & $\mathrm{~ns}$ \\
LDL cholesterol $(\mathrm{mg} / \mathrm{dl})$ & $124.3 \pm 35.8$ & $101.6 \pm 39.2$ & 0.003 \\
Triglycerides $(\mathrm{mg} / \mathrm{dl})$ & $288.1 \pm 125.7$ & $195.1 \pm 80$ & 0.01 \\
\hline
\end{tabular}

\section{Conclusion}

$\Rightarrow$ In obese patients with T2DM, once-weekly exenatide increases metabolic control, decreases body weight and improves lipid profile. However, once-weekly exenatide does not exert beneficial effects on blood pressure.

$\Rightarrow$ Age, sex, duration of diabetes, baseline HbA1c, and BMI were not predictors of efficacy of once-weekly exenatide.

\begin{tabular}{|c|c|c|c|c|c|}
\hline \multirow[t]{2}{*}{ ب $483-$-EP } & & & \multirow{2}{*}{$\begin{aligned} & \text { Poster } \text { ECE2015 2015 } \\
& \text { presented } \\
& \text { at: }\end{aligned}$} & \multirow{2}{*}{ 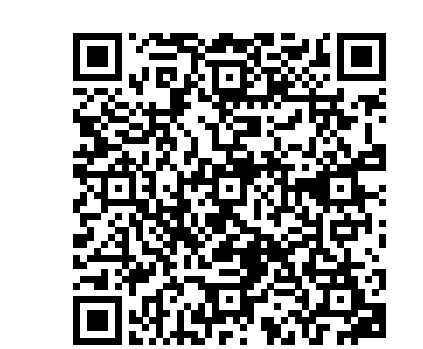 } & \multirow{2}{*}{$\begin{array}{l}\text { Pogter } \\
\text { Sessiononline }\end{array}$} \\
\hline & Jose Carlos Fernandez-Garcia & DOI: 10.3252/pso.eu.17飞ece.2015 & & & \\
\hline
\end{tabular}

